\title{
Aplicação da Realidade Aumentada ao ensino e aprendizagem do campo magnético de um ímã em forma cilíndrica e em condutor retilíneo
}

\author{
Adriana Azeredo de Souza Ribeiro - IFF - adriasribeiro@yahoo.com.br \\ Renata Lacerda Caldas - IFF - renata.caldas@iff.edu.br \\ Suzana da Hora Macedo - IFF - shmacedo@iff.edu.br
}

Resumo: Este artigo apresenta uma proposta de ensino e aprendizagem de campo magnético apoiada por objetos de aprendizagem desenvolvidos em ambiente de Realidade Aumentada. Para isso, foram utilizados dois objetos, um ímã em forma cilíndrica e em um fio condutor retilíneo, onde foi visualizada a simulação da representação das linhas de campo magnético em três dimensões usando Realidade Aumentada. Participaram da atividade alunos do terceiro ano do ensino médio de uma escola particular. Nos resultados obtidos, através das representações pictóricas, verificouse nos modelos mentais que os alunos conseguiram reconciliar os conceitos e inferiram ideias à construção do conceito de campo magnético em três dimensões (3D), e ao visualizar as linhas de campo magnético e obter uma melhor compreensão do conceito físico estudado. Sendo assim, este estudo contribuiu para uma aquisição de significados e apontando para indícios de uma Aprendizagem Significativa e consequentemente, para a melhoria do ensino de Física, trazendo subsídios tanto para professores quanto para alunos.

Palavras-chave: Realidade Aumentada, ímã em forma cilíndrica, condutor retilíneo, campo magnético, Aprendizagem Significativa.

\section{Application of Augmented Reality to teaching and learning the magnetic field of a magnet in a cylindrical shape and in a straight conductor}

\begin{abstract}
This article presents a proposal for magnetic field teaching and learning supported by learning objects developed in an Augmented Reality environment. For that, two objects were used, a magnet in a cylindrical shape and in a straight conductive wire, where the simulation of the representation of the magnetic field lines in three dimensions was visualized using Augmented Reality. Third year high school students from a private school participated in the activity. In the obtained results, through the pictorial representations, it was verified in the mental models that the students managed to reconcile the concepts and inferred ideas to the construction of the magnetic field concept in three dimensions (3D), and when visualizing the magnetic field lines and obtaining a better understanding of the studied physical concept. Thus, this study contributed to an acquisition of meanings and pointing to signs of a Meaningful Learning and, consequently, to the improvement of Physics teaching, bringing subsidies for both teachers and students.
\end{abstract}

Keywords: Augmented Reality, magnet in cylindrical shape, straight conductor, magnetic field, Meaningful Learning.

\section{Introdução}

É possível perceber que os indivíduos possuem dificuldades de representar o que captam no mundo externo. No contexto escolar, os alunos elaboram representações pessoais para compreender as informações captadas nas aulas. 
A teoria dos modelos mentais (MM) de Johnson Laird (1983) enfatiza que essa captação não cria um modelo físico a ser visualizado, isto é, que as representações mentais são maneiras de representar internamente o mundo externo. Segundo esse autor, as pessoas não captam o mundo exterior diretamente, mas constroem representações mentais desse mundo (MOREIRA, 2011).

$\mathrm{Na}$ escola este contexto não é diferente quanto a conceitos a serem estudados de forma abstrata, oferecendo, portanto, dificuldades de compreensão devido a sua abstração. O conceito físico de campo magnético é um exemplo claro desta dificuldade de representação.

As dificuldades de aprendizagem apresentadas pelos alunos no estudo do Eletromagnetismo estão relacionadas com a ideia/noção de campo eletromagnético, uma vez que este campo não é visível e dificilmente o aluno terá algum conhecimento prévio a respeito deste fenômeno e as formulações matemáticas que descrevem os fenômenos relacionados ao Eletromagnetismo são de difícil compreensão (TRES; SANTOS, 2016).

Paz afirma que (2007, p. 15), "as dificuldades de aprendizagem dos conteúdos de Eletromagnetismo se concentram no entendimento das interações e comportamento das variáveis eletromagnéticas no espaço tridimensional". Pesquisas (RICARDO; FREIRE, 2007) apontam que tais dificuldades são encontradas tanto em alunos do ensino médio (EM) quanto em alunos do ensino superior (ES), devido à abstração envolvida (PAZ, 2007).

Portanto, a importância do estudo do conceito de campo magnético foi tratada nesta pesquisa em nível médio, por ser um tema de alto nível de abstração no que diz respeito a sua representação espacial e por oferecer relevante dificuldade na visualização dos fenômenos eletromagnéticos associados à influência do campo magnético.

O estudo deste conceito para os alunos da Educação Básica, em especial os do EM, principalmente para aqueles que querem ingressar nos cursos técnicos, é previsto no contexto do artigo $36 \S^{\circ}$ da Lei de Diretrizes e Bases da Educação (LDB), que estabelece que ao final do curso o aluno deva ter o domínio dos princípios científicos e tecnológicos que norteiam a produção moderna (BRASIL, 2013).

De acordo com os Parâmetros Curriculares Nacionais (PCN's, 2002), os objetivos do ensino médio em cada área de conhecimento devem envolver, de forma combinada, o desenvolvimento de conhecimentos práticos, contextualizados, que respondam às necessidades da vida contemporânea e o desenvolvimento de conhecimentos mais amplos e abstratos, que correspondam a uma cultura geral e a uma visão de mundo (BRASIL, 2002).

O processo de ensino que busca a consolidação do conhecimento de forma significativa deve visar uma aprendizagem significativa com a utilização de estratégias diferenciadas que são de extrema importância para que cada aluno construa as relações necessárias à sala de aula e suas vivências.

Para desenvolver esta pesquisa buscou-se fundamentação na Teoria da Aprendizagem Significativa (AS) de David Ausubel (1963). Para que ocorra a aprendizagem do aluno é muito importante valorizar aquilo que o aluno já sabe, para que nele a informação se ancore, adquira significados e resulte na Aprendizagem Significativa (AUSUBEL, 1963, p. 6).

No caso da Física, os PCN's recomendam que a mesma deva apresentar-se como um conjunto de competências específicas que permitam perceber e lidar com os fenômenos naturais e tecnológicos, presentes tanto no cotidiano mais imediato quanto na compreensão do universo distante, a partir de princípios, leis e modelos por ela 
construídos. Embora nos PCN's seja reconhecido que não existem fórmulas prontas para traduzir suas diretrizes em práticas escolares concretas, nestes mesmos documentos são apontadas algumas estratégias que deveriam ser implementadas pelos professores ao se pensar no ensino de Física (BRASIL, 2002).

Neste contexto, a Lei de Diretrizes e Bases (BRASIL, 2013) reforça a importância do avanço do estudo relacionando a ciência e suas tecnologias, o que justifica a relevância de um ensino focado na aplicação de recursos tecnológicos como auxílio do estudo sobre o eletromagnetismo.

Mediante o contexto até aqui apresentado, este trabalho é guiado pela seguinte questão: em que medida uma intervenção didática com o apoio tecnológico do ambiente em RA pode contribuir para explicitação dos MM envolvendo o Campo Magnético, em nível médio?

Portanto, o objetivo deste artigo é investigar, através da intervenção didática (atividade experimental), ancorada ao ambiente tecnológico RA, uma melhor representação mental do campo magnético para o ensino médio. A externalização das representações das linhas de campo magnético pelos alunos será analisada do ponto de vista da Teoria dos MM de Johnson-Laird (1983), na qual os modelos mentais são representações analógicas, um tanto quanto abstraídas, de conceitos, objetos ou eventos que são espacial e temporalmente análogos a impressões sensoriais, mas que podem ser vistos de qualquer ângulo e que, em geral, não retêm aspectos distintivos de uma dada instância de um objeto ou evento (STEMBERG, 1996, citada por MOREIRA, 2011).

Este artigo está organizado da seguinte forma: após esta introdução é conceituado o ambiente de RA e seu potencial na área da educação. Em seguida, são apresentados os materiais e métodos utilizados na construção da atividade experimental e os objetos de aprendizagem (OA) do ímã em forma cilíndrica e em condutor retilíneo. Na sequência, são apresentados os resultados e as discussões sobre a atividade experimental e o recurso tecnológico em ambiente RA por partes dos alunos, concluindo com as considerações finais e referências utilizadas neste estudo.

\section{Fundamentação Teórica}

O conceito de RA de acordo com Kirner (2008, p. 4) deu-se no início dos anos 1990. Segundo o autor, é definida pela inserção de objetos virtuais no ambiente físico, mostrada ao usuário, em tempo real, com o apoio de algum dispositivo tecnológico, usando a interface do ambiente real, adaptada para visualizar e manipular os objetos reais e virtuais (KIRNER, 2008, p. 9). Azuma (1997) define a Realidade Aumentada como um sistema que: combina elementos virtuais com o ambiente real; é interativa e tem processamento em tempo real; é concebida em 3D (AZUMA, 1997).

Essa tecnologia vem sendo utilizada de maneira crescente em diferentes áreas. Há diversos estudos e aplicações desenvolvidas sobre ela, que tem colaborado como ferramenta de apoio a educadores e estudantes no desenvolvimento dos processos de ensino e da aprendizagem (FRANKLIN et al., 2011).

Partindo do benefício dessa tecnologia (BUCHAU et al. (2009) construíram três aplicações baseadas em RA para serem utilizadas na visualização do campo magnético de um ímã, do campo magnético de um solenoide e do campo magnético de uma antena e estas aplicações permitem que se visualize os campos magnéticos em três dimensões.

Para Lemos e Carvalho (2010, p. 2), o software educativo "[...] pode ser a interface entre os professores e os estudantes como uma ferramenta auxiliar para melhorar o processo de ensino e de aprendizagem de um conteúdo ou assunto educacional...". E 
segundo Wiley (2000), OA é qualquer recurso digital que pode ser reutilizado para apoiar a aprendizagem (LEMOS; CARVALHO, 2010), (WILEY, 2000).

Assim, com o OA o aluno poderá visualizar a interação do campo magnético e interagir com o mesmo, como nunca experimentado anteriormente, levando o estudante ao despertar para novos conhecimentos com o apoio da RA.

Para o uso da RA são necessários alguns requisitos básicos como: um computador, uma webcam, um programa desenvolvido com uma biblioteca de programação e uma placa de papel com um símbolo no interior, denominado marcador fiducial. Este marcador é colocado em um objeto no qual se deseja que ocorra a interação. Ao ser visualizado pela câmera do computador, o marcador deve ser reconhecido e levará a uma biblioteca de programação pré-estabelecida. Aparecerá, então, na tela do computador, o primeiro objeto em que estava o marcador, juntamente com o objeto que estará na biblioteca. Os dois objetos serão fundidos em um mundo misto que misturará o mundo real com o mundo virtual (MACEDO et al., 2011, p. 158).

A tecnologia de RA, ao misturar objetos virtuais ao mundo real, conta além de (câmera) computadores também com (câmera) dos dispositivos móveis, como tablets e smartphones, para capturar e mostrar a interação, permitindo ao usuário visualizar e interagir com um objeto tridimensional (HOUNSELL, TORI, KIRNER, 2018).

Acompanhando a tendência do desenvolvimento tecnológico no que se refere aos dispositivos móveis, em especial para celulares, aliado ao aumento da capacidade de processamento desses dispositivos, os smartphones, a RA tende a acompanhar tal evolução ficando cada vez mais popular nesses dispositivos.

O funcionamento da RA a partir do smartphone, se dá da seguinte maneira: um marcador impresso (que "contém" objeto virtual) é capturado pela câmera do smartphone, que por meio de um aplicativo percebe e rastreia o marcador, projetando o objeto virtual tridimensional, podendo assim ser visualizado pelo usuário pela tela do dispositivo (MACEDO; SILVA; BURIOL, 2016).

A RA vem sendo utilizada de maneira crescente em diferentes áreas. Há diversos estudos e aplicações desenvolvidas sobre ela, que tem colaborado como ferramenta de apoio a educadores e estudantes no desenvolvimento dos processos de ensino e da aprendizagem (FRANKLIN et al., 2011).

Essa tecnologia caracteriza-se como uma técnica que, utilizando de algoritmos de visão computacional, tem por finalidade sobrepor informações virtuais - textuais ou gráficas - em reais em tempo real, permitindo que haja uma melhor percepção e interação do usuário com esse ambiente (JOHNSON et al., 2011). Nesse sentido, Johnson e colaboradores (2011) afirmam que a RA deverá causar grande impacto na educação superior nos próximos anos (JOHNSON et al., 2011).

A proposta de ensino utilizando o recurso de RA, inspirada na teoria da Aprendizagem Significativa de David Ausubel, leva em conta os conhecimentos prévios dos alunos sobre o conceito de campo magnético, para uma aprendizagem com significados, compreensão, capacidade de explicar, de aplicar o conhecimento adquirido em novas situações.

Moreira (2006, p. 13-14) apresentando uma visão geral da teoria da AS, afirma que, para Ausubel, a aprendizagem, tal como ela ocorre na sala de aula, é muito influenciada por aquilo que o aluno já sabe. Nesta perspectiva, Aprendizagem Significativa, é o processo por meio do qual uma nova informação relaciona-se com um aspecto relevante da estrutura de conhecimento do indivíduo e ocorre quando a nova informação se ancora em conceitos ou proposições relevantes pré-existentes na estrutura 
cognitiva do mesmo. Esta estrutura é definida por Ausubel como elemento subsunçor.

Os conceitos intuitivos de força e campo já existem na estrutura cognitiva do aluno e servirão de subsunçores para as novas informações sobre certos tipos de força e campo. No entanto, o processo de ancoragem da nova informação resulta em crescimento e modificação do subsunçor. Os subsunçores existentes na estrutura cognitiva do aluno podem ser abrangentes e bem desenvolvidos ou limitados e pouco desenvolvidos.

Em contraste com a AS, Ausubel menciona que a aprendizagem mecânica ou automática ocorre até que alguns conhecimentos relevantes às novas informações da mesma área existam na estrutura cognitiva e possam servir de facilitadores, ainda que pouco trabalhados. À medida que a aprendizagem começa a ser significativa, esses facilitadores vão sendo assimilados, tornando-se, pois, mais capazes de facilitar ou ancorar a nova informação (MOREIRA, 2006, p. 15-17).

\section{Materiais e Métodos}

A metodologia utilizada no presente trabalho foi dividida em três etapas. A primeira se constituiu na realização da atividade intitulada Linha de Campo Magnético a obtenção dos MM do Campo Magnético em ímã em formata cilíndrica e no condutor retilíneo. A segunda etapa constituiu na aplicação da atividade experimental dos objetos de aprendizagem RA para inferir a ideia do campo magnético tridimensional. Na terceira etapa, o foco foi avaliar a RA do ponto de vista dos alunos. As 3 etapas ocorreram sequencialmente com duração de $2 \mathrm{~h} /$ aula.

Etapa 1: Para atender o foco deste trabalho, que é a aprendizagem do campo magnético, foi solicitado que os alunos representassem as linhas de indução nos dois casos propostos, com o intuito de fazer o levantamento dos conhecimentos prévios quanto ao comportamento tridimensional das linhas de indução, com base no estudo de eletromagnetismo em aulas anteriores. Esta etapa teve uma duração de $20 \mathrm{~min}$.

Etapa 2: Deu-se continuidade com a apresentação do recurso tecnológico RA para cada caso (Figuras 1 e 2), aconselhando interações entre aluno, professor e material educativo (GOWIN, 1981), tornando a aula mais atrativa e dinâmica (MOREIRA, 2011). Após a visualização das linhas de campo magnético em três dimensões, nos dois casos com o uso do recurso tecnológico RA, solicitou-se que os alunos confrontassem suas representações pictóricas com a figura na tela do computador, advinda do uso da RA, objetivando frente a uma nova situação inferir novas informações em seus MM. Esta etapa teve duração de 50 min.

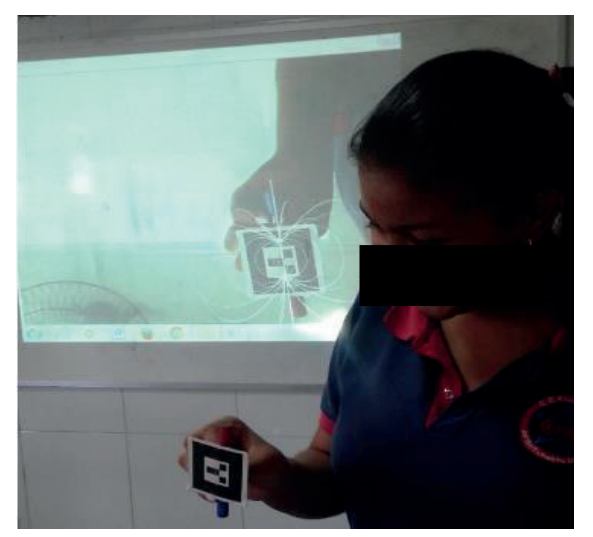

Figura 1 - Objeto em RA com as respectivas linhas de campo magnético e os marcadores fiduciais (ímã de forma cilíndrica e as linhas de campo magnético)

Fonte: autores 


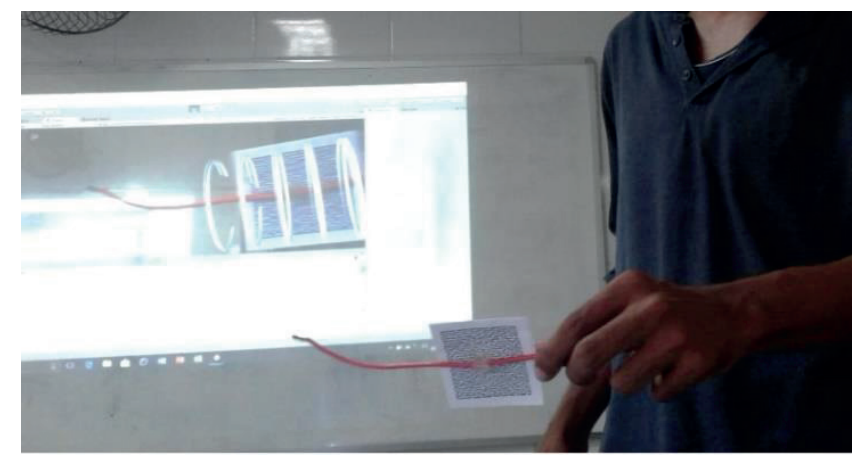

Figura 2 - Objeto em RA com as respectivas linhas de campo magnético e os marcadores fiduciais (condutor retilíneo)

Fonte: autores

Para a criação do ambiente em RA do OA conforme a Figura 1, foi utilizado o Artoolkit, que funciona em qualquer computador. É uma biblioteca de código aberto e gratuita, a qual permite programadores desenvolverem facilmente aplicações de Realidade Aumentada (MACEDO, 2011). Com isso, foi utilizado um objeto real, um ímã em forma cilíndrica, e o marcador para o respectivo objeto, sendo que este OA já havia sido desenvolvido anteriormente por uma das autoras.

Para a criação do ambiente em RA do OA, conforme a Figura 2, foi utilizado o Unity (3D), que é um ambiente de desenvolvimento integrado (IDE), voltado especificamente para o desenvolvimento de jogos e de visualização 3D (MORAIS et al., 2017). O VUforia é uma plataforma mais amplamente utilizada para o desenvolvimento de RA, com suporte para os principais telefones, tablets e óculos. Os desenvolvedores podem facilmente adicionar funcionalidade avançada de visão computacional aos aplicativos Android, iOS e UWP, para criar experiências de recuperação da realidade virtual que interagem realisticamente com objetos e o ambiente (VUFORIA, 2017). Assim, o Vuforia foi usado para trabalhar com RA, pois é um kit de desenvolvimento de software que pode ser usado para criar aplicativos de realidade aumentada integrado com o Unity 3D. No experimento utilizando o OA da Figura 2 também foi utilizado um objeto real, um condutor retilíneo, e o marcador para o respectivo objeto (MORAIS et al., 2017). Este OA foi desenvolvido e utilizado anteriormente em um dos trabalhos das autoras.

Etapa 3: Foi aplicado um questionário contendo seis perguntas referentes à avaliação do uso do recurso tecnológico RA no estudo do campo magnético. Esta etapa teve duração de 30 min.

Os instrumentos para a coleta de dados buscaram investigar a aprendizagem do aluno quanto ao aspecto tridimensional do campo magnético por meio das representações. Seis alunos do terceiro ano do EM da rede particular de ensino participaram efetivamente das três etapas, identificados cada um por uma letra do alfabeto.

A análise dos resultados, baseada no estudo dos modelos mentais de trabalho (MMt), representações mentais analógicas (combinações por meio visual) e/ou proposicionais (a mente capta uma ideia), será apresentada a seguir.

\section{Resultados e Discussão}

Após a conclusão das três etapas, foram levantados os resultados da atividade com a representação das linhas de campo magnético em busca de significados quanto ao comportamento Tridimensional do Campo Magnético e sua visualização com o uso da 
RA objetivando a consolidação do conhecimento adquirido pelos alunos ao tema.

A Figura 3 apresenta as representações pictóricas dos seis alunos (A, B, C, E, F e G) para o caso do ímã em formato cilíndrico.

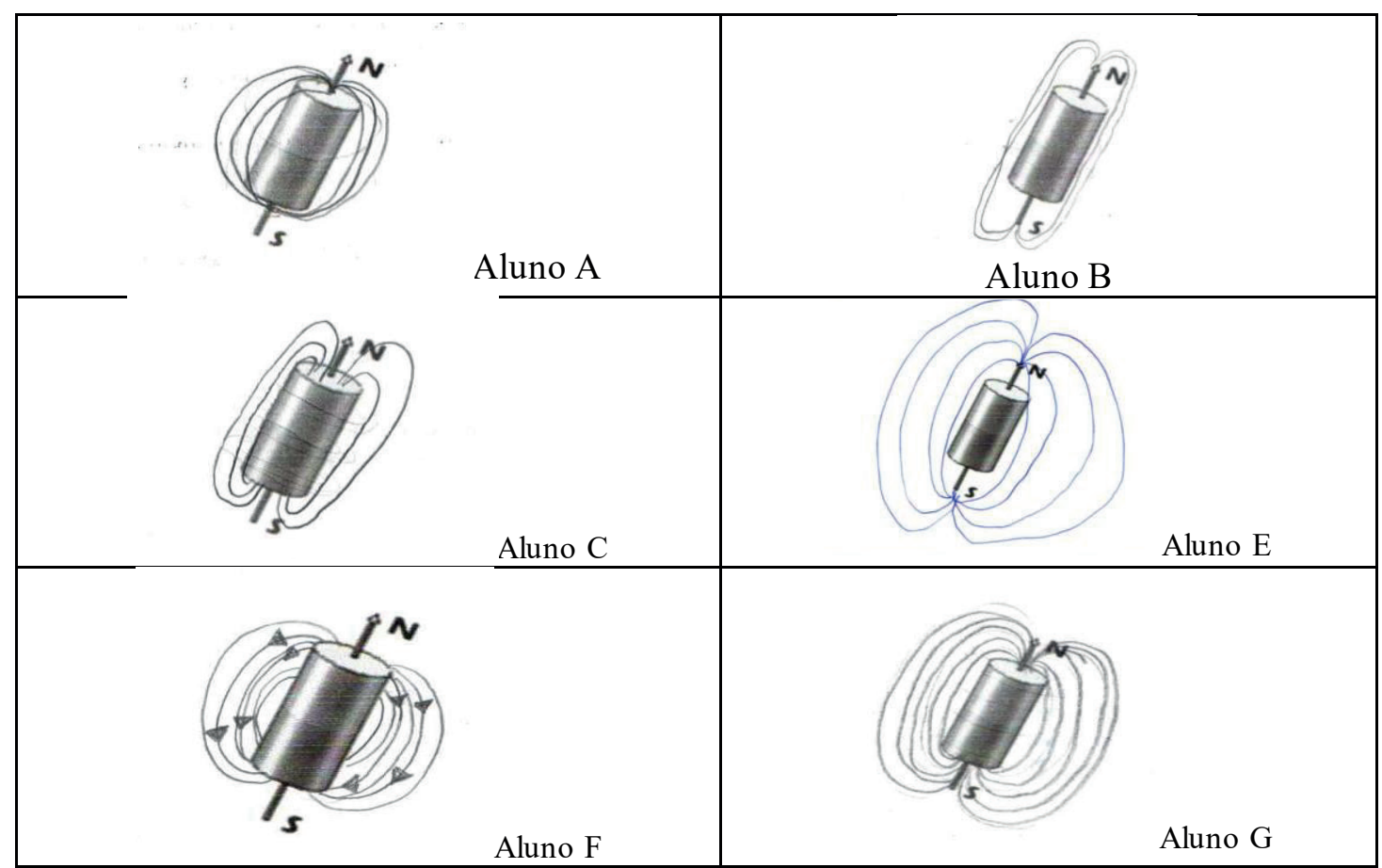

Figura 3 - Primeiro caso da atividade linhas de campo magnético

Fonte: autores

As representações pictóricas apresentadas por todos os alunos apresentaram de maneira parcialmente correta as linhas de campo magnético do ímã em forma cilíndrica, embora alguns destes tenham inserido também linhas diferenciadas em suas representações.

Somente o aluno $\mathrm{F}$ indicou setas em sua representação referenciando a grandeza vetorial do campo magnético. Mas os alunos $A, B, C$, e $G$, apresentaram em suas representações linhas mais finas ao redor do ímã, explicitando a concepção de campo tridimensional, destacando a representação do aluno $G$, com as linhas de campo mais densas. As representações elaboradas refletem MMt sobre campo magnético para este caso.

Na Figura 4 tem-se as representações pictóricas representadas dos seis alunos (A, $\mathrm{B}, \mathrm{C}, \mathrm{E}, \mathrm{F}$ e G) para o caso do condutor retilíneo.

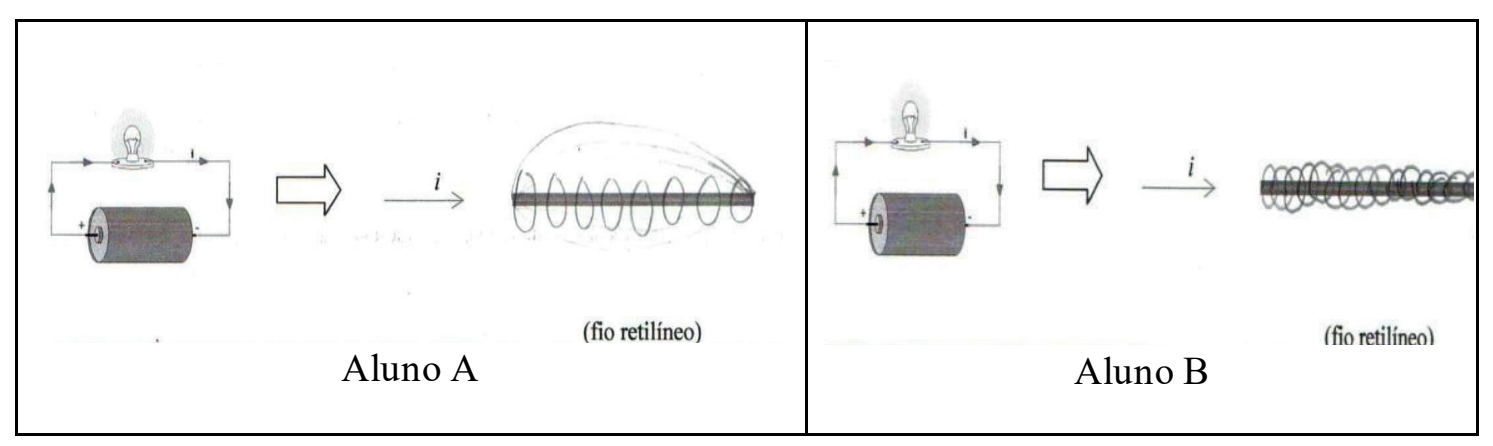




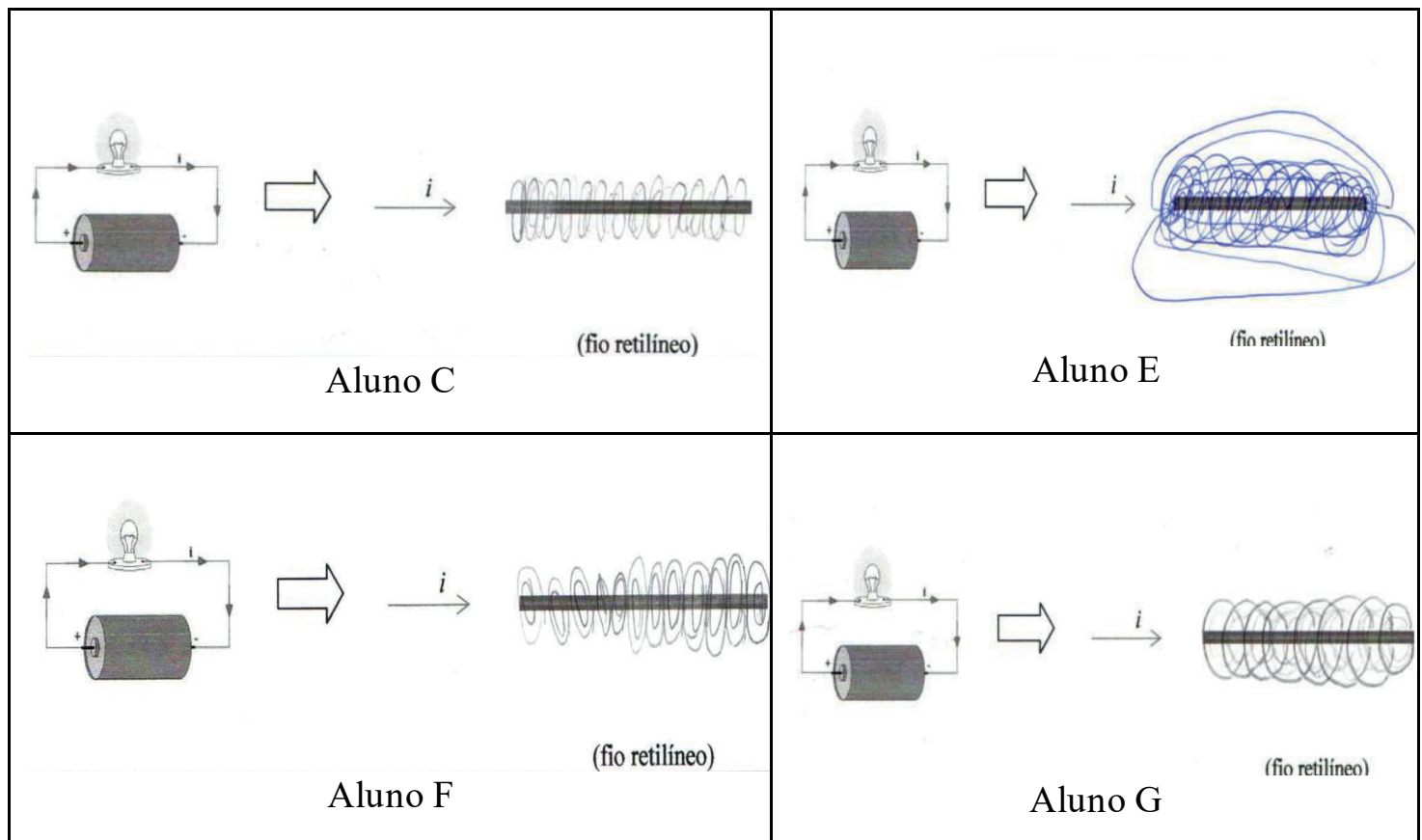

Figura 4 - Segundo caso da atividade linhas de campo magnético

Fonte: autores

Verifica-se que os seis alunos explicitaram as suas representações pictóricas com linhas em volta do fio condutor, mas os alunos A e E, apresentaram linhas em todas as direções e o aluno $\mathrm{G}$ com linhas mais densas, indicando a ideia de campo tridimensional. As representações elaboradas configuram MMt sobre campo magnético para este caso.

Consequentemente, pode-se perceber com a aplicação dessa atividade com o uso do recurso tecnológico RA, o papel fundamental desta estratégia para a compreensão sobre a forma de propagação do campo magnético. O fato de os alunos conseguirem visualizar as linhas de campo, apesar de explicitarem bidimensionalmente, apontou satisfatoriamente que o campo magnético é uma grandeza tridimensional, também foi possível experimentar a curiosidade e o entusiasmo dos alunos quanto a utilização do recurso tecnológico RA. Logo, os alunos puderam confrontar seus MMt sobre a ideia de campo magnético nos dois casos. Todos queriam experimentar a RA, tornando a aula mais atrativa e dinâmica.

O Gráfico 1 mostra as seis perguntas propostas no questionário e as respostas dos alunos sobre do uso do recurso tecnológico RA no estudo do campo magnético. 


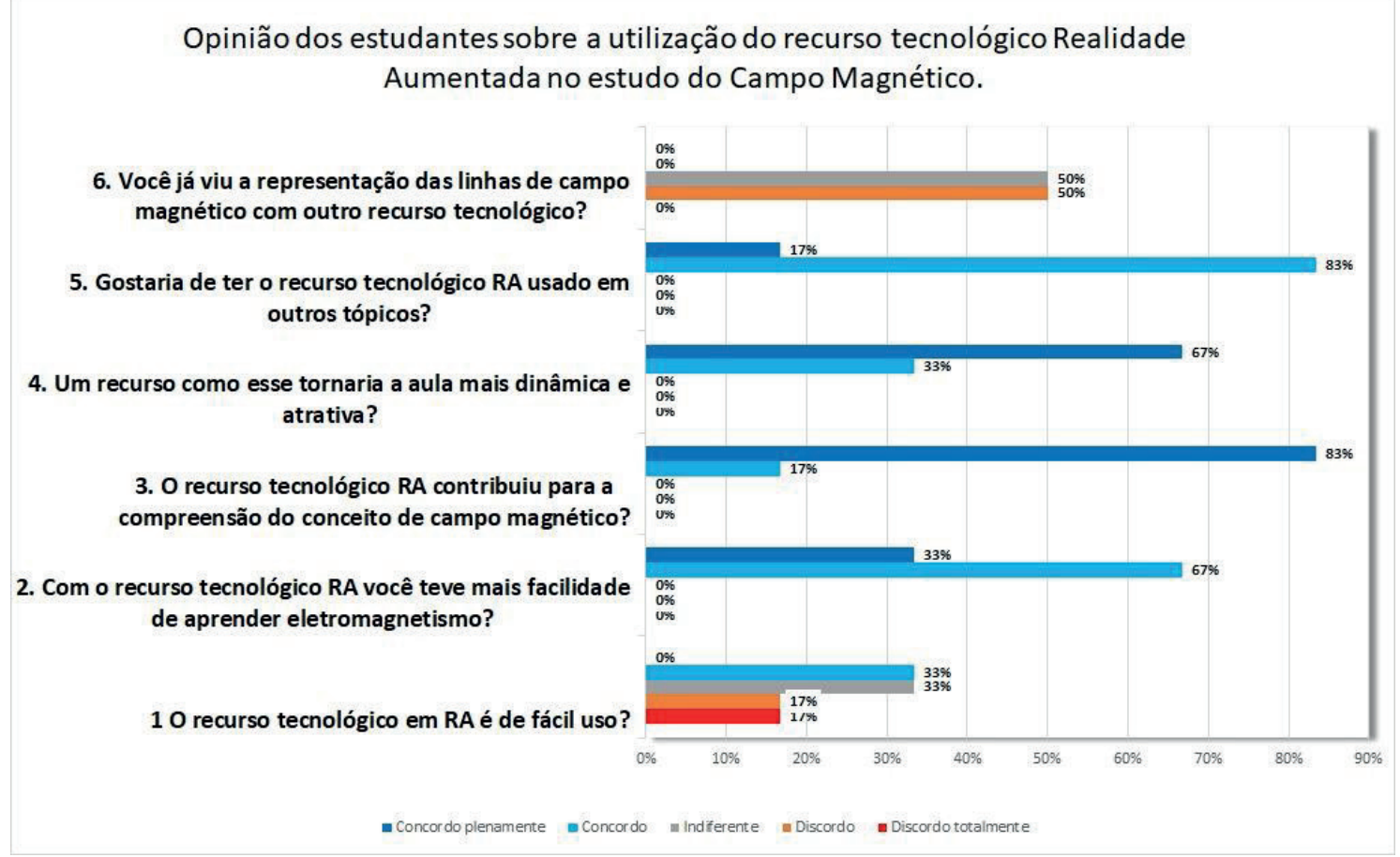

Gráfico 1 - Gráfico sobre o uso da RA no estudo do campo magnético Fonte: autores

Com relação à pergunta 1 , se o recurso tecnológico foi de fácil uso, $33 \%$ dos alunos concordam. Na pergunta 2 , se com o recurso tecnológico RA teve mais facilidade de aprender eletromagnetismo, 67\% dos alunos concordaram. Na pergunta 3, se o recurso tecnológico RA contribuiu para a compreensão do conceito de campo magnético, $83 \%$ dos alunos concordaram plenamente. Na pergunta 4 , se recursos como esse tornam a aula mais dinâmica, $67 \%$ dos alunos concordaram plenamente. Na pergunta 5 , se gostariam de ter o recurso tecnológico RA usado em outros tópicos, $85 \%$ concordaram e na pergunta 6, se já viram a representação das linhas de campo magnético com outro recurso tecnológico, $50 \%$ dos alunos discordam e, também, $50 \%$ consideraram indiferente.

As respostas dos alunos mostram que o recurso tecnológico em RA, além de tornar a mais interativa e atraente, possibilitou o vislumbre das diferentes linhas de campo magnético em três dimensões do ímã em forma cilíndrica e no condutor retilíneo. Os experimentos em RA ajudaram a entender o fenômeno que nunca tinham visto, esclareceu dúvidas, adquiriram mais conhecimentos e todos gostariam de ter esse recurso tecnológico em outras tópicos, além de nunca terem visto as linhas de campo magnético com outras tecnologias.

Nesse sentido, diversos estudos comprovam que a utilização da RA traz contribuições para a percepção e motivação dos usuários em diversas atividades no contexto educacional, auxiliando na aprendizagem em diversas áreas do conhecimento (AYER et al., 2016).

\section{Considerações Finais}

Este trabalho sugere o recurso tecnológico em RA para ser utilizado por professores de Física do Ensino Médio proporcionando a compreensão tridimensional do Campo Magnético através da visualização em três dimensões por parte dos alunos.

Foram utilizados dois OA que se apresentam em auxiliar a percepção do aluno na 
construção e consolidação das linhas de campo magnético de um ímã de forma cilíndrica e em condutor retilíneo em 3D.

Com base nas respostas dos alunos, a RA mostrou ser relevante para o uso em sala de aula no ensino do campo magnético devido à facilidade de interação e visualização do mundo misturado, e pode ser usado várias vezes proporcionando uma aprendizagem significativa com a visualização de situações-problema não vivenciadas no cotidiano do aluno, de maneira mais natural e intuitiva sem necessidade de treinamento ou adaptação, proporcionando assim modelos mentais mais elaborados, ou seja, agregando novos conceitos na compreensão de campo, fato atribuído certamente ao uso da RA e fazendo com que essa tecnologia seja bastante apropriada para trabalhos colaborativos e remotos.

\section{Referências}

AUSUBEL, D. P. The psychology of meaningful verbal learning. New York: Grune and Stratton, 1963.

AYER, S. K.; MESSNER, J. I.; ANUMBA, C.J. Augmented Reality Gaming in Sustainable Design Education. Journal of Architectural Engineering, 2016, v. 22, n. 1, p. 1-9.

AZUMA, T. R. A Survey of Augmented Reality. Hughes Research Laboratories 3011 Malibu Canyon Road, MSRL96, Malibu, 1997. Disponível em: https://www.cs.unc.edu/ azuma/ARpresence.pdf. Acessado em 16 jul. 2017.

BRASIL. Ministério da Educação. Secretaria de Educação Média e Tecnológica. Lei de Diretrizes e Bases da Educação. Brasília: MEC/SEMTEC, 2013.

BRASIL. Ministério da Educação. Secretaria da Educação Média e Tecnológica. Parâmetros Curriculares Nacionais $+(\mathrm{PCN}+)$ - Ciências da Natureza e suas Tecnologias. Brasília: MEC, 2002.

BUCHAU, ANDRÉ; RUCKER, WOLFGANG M.; WÖSSNER, UWE; BECKER, MARTIN. Augmented Reality in Teaching Eletrodynamics. The International Journal for Computation and Mathematics in Electrical and Electronic Engineering. Vol. 28 No. 4. 2009. pp 948-963.

FRANKLIN, F.; BORGES, A. C.; SANTOS, E. C. B. Um Sistema Interativo Com Realidade Aumentada Como Ferramenta De Ensino-Aprendizagem em Diabetes Mellitus. In: SBIE, SIMPÓSIO BRASILEIRO DE INFORMÁTICA NA EDUCAÇÃO, 22, 2011, Aracaju-SE. Anais. Disponível em: <http://www.brie.org/index.php/anaissbie>. Acesso em: 19 dez. 2013.

GOWIN, D.B. Educating. Ithaca, NY, Cornell University Press, 1981.

HOUNSELL, M. S (org); TORI, R.; KIRNER. Introdução a Realidade Virtual e Aumentada. Porto Alegre: Editora SBC, 2018.

JOHNSON, L. SMITH, R., WILLIS, H., LEVINE, A., and HAYWOOD, K. The 2011 Horizon Report. The New Media Consortium, Austin, Texas, Rel. Téc., 2011.

JOHNSON-LAIRD, P. Mental Models. Cambridge, MA: Harvard University Press, 1983.

KIRNER, C. Definições. In: SISCOUTO, R; COSTA, R. (orgs.). Realidade virtual e aumentada: uma abordagem tecnológica, 2008. Disponível em: <http://www.ckirner. com/download/livros/RVA08-Livro.zip>. Acesso em: 20 agosto. 2013.

LEMOS, B. M., CARVALHO, C. V. Uso da Realidade Aumentada para Apoio ao Entendimento da Relação de Euler. RENOTE. In: Revista Novas Tecnologias na Educação. V. 8, p. 1-10, 2010.

MACEDO, A. C.; SILVA, J. A.; BURIOL, T. M. Usando Smartphone e Realidade 
aumentada para estudar Geometria espacial. Revista Novas Tecnologias (RENOTE), Rio Grande do Sul, v.14, n. 2, p. 10, dez. 2016. Disponível em: http://www.seer.ufrgs.br/index.php/renote/article/view/70688/40123. Acesso em: 16 dez. 2020.

MACEDO, S. H.; BIAZUS, M. C. V.; FERNANDES, F. A. Ensino do campo magnético de um ímã em forma de barra utilizando recursos de realidade aumentada. Informática na Educação: teoria e prática, Porto Alegre, v. 14, n. 1, p. 158, jan./jun. 2011.

MORAIS, C.G. B; Silva, C. R. S; Mendonça, A. K. S. Utilização de dispositivo móvel com Realidade Aumentada: um estudo de caso na Educação Infantil com o aplicativo Cubo Kids. In: VI CONGRESSO BRASILEIRO DE INFORMÁTICA NA EDUCAÇÃO, Mossoró, 2017.

MOREIRA, M. A. Aprendizagem Significativa um Conceito Subjacente. Psicologia da Educação, 2011.

MOREIRA, M. A. A teoria da aprendizagem significativa e sua implementação em sala de aula. Brasília: Editora da UnB, 2006.

PAZ, A. M. Atividades Experimentais e Informatizadas: Contribuições para o Ensino de Eletromagnetismo (2007), Universidade Federal de Santa Catarina, Florianópolis, 228 p. Tese de Doutorado.

RICARDO. C. E.; FREIRE. J.C.A. A concepção dos alunos sobre a física do ensino médio: um estudo exploratório. (The students' conceptions about high school's physics subject: an exploratory study). Revista Brasileira de Ensino de Física, v. 29, n. 2, p. 251-266, (2007). Disponível em: http://www.scielo.br/pdf/rbef/v29n2/a10v29n2. Acesso: 13 mar 2017.

TRES, C.; SANTOS, S. A. Estratégias Diversificadas no Ensino do Eletromagnetismo para Facilitar a Aprendizagem Significativa. Os Desafios da Escola Pública Paranaense na Perspectiva do Professor PDE, v. 1, 2016.

WILEY, D. A. (2000). Connecting learning objects to instructional design theory: A definition, a metaphor, and a taxonomy. In: D. A. WILE (ED.), THE INSTRUCTIONAL USE OF LEARNING OBJECTOS: Online Version. Retrieved MONTH DAY, YEAR, from the World WideWeb: http://reusability.org/read/chapters/wiley.doc. Acesso: 13 mar 2017. 